\title{
Jacquette on Grelling's Paradox
}

\section{JEFFREY KETLAND}

Dale Jacquette (Jacquette 2004) argues that, despite usual arguments to the contrary, a version of Grelling's paradox reappears in type theory, and thus that type theory, or higher-order logic, is inconsistent:

I maintain in what follows that type theory, although it appears tailor-made to solve the problem, does not actually forestall Grelling's paradox, but that the paradox remains derivable in spite of rigorously enforced type restrictions. (Jacquette 2004: 251)

Jacquette's argument is based on the following 'definitions':

(1) $\forall F^{n+1} \forall F^{n}\left(H^{n+1}\left(F^{n}\right) \leftrightarrow \sim F^{n+1}\left(F^{n}\right)\right)$

(2) $\forall F^{n} \forall F^{n-1}\left(H^{n}\left(F^{n-1}\right) \leftrightarrow \sim F^{n}\left(F^{n-1}\right)\right)$.

Definition (1) is meant to define a type $n+1$ heterologicality relation $H^{n+1}$ such that, for any type $n+1$ relation $F^{n+1}$ and type $n$ relation $F^{n}$, $H^{n+1}$ applies to $F^{n}$ just in case $F^{n+1}$ does not apply to $F^{n}$. Definition (2) is meant to define a type $n$ heterologicality relation $H^{n}$ such that, for any type $n$ relation $F^{n}$ and type $n-1$ relation $F^{n-1}, H^{n}$ applies to $F^{n-1}$ just in case $F^{n}$ does not apply to $F^{n-1}$.

The argument proceeds by instantiating the second quantifier in (1) with ' $H^{n}$ ', thus,

(3) $\forall F^{n+1}\left(H^{n+1}\left(H^{n}\right) \leftrightarrow \sim F^{n+1}\left(H^{n}\right)\right)$.

Next, instantiate the variable ' $F^{n+1}$ ' with ' $H^{n+1}$, giving the contradiction,

(4) $H^{n+1}\left(H^{n}\right) \leftrightarrow \sim H^{n+1}\left(H^{n}\right)$.

To see what is wrong with Jacquette's argument, reconsider the 'definition' (1). In writing the argument dependency (i.e. the free variables which occur) of the definiendum ' $H^{n+1}$ ' as ' $H^{n+1}\left(F^{n}\right)$ ', Jacquette wishes to represent $H^{n+1}$ as a one-place relation. But the definiens is ' $\sim F^{n+1}\left(F^{n}\right)$ ' and thus contains two distinct free variables, ' $F^{n+1}$ ' and ' $F^{n}$ '. So, $H^{n+1}$ must be a two-place relation. That is, the definition (1) is meant to fix $H^{n+1}$ as applying to a pair $\left(F^{n+1}, F^{n}\right)$ just in case $F^{n+1}$ does not apply to $F^{n}$. The free variables should thus be written ' $H^{n+1}\left(F^{n+1}, F^{n}\right)$ '. So, correctly expressed, definition (1) should read,

(5) $\forall F^{n+1} \forall F^{n}\left(H^{n+1}\left(F^{n+1}, F^{n}\right) \leftrightarrow \sim F^{n+1}\left(F^{n}\right)\right)$.

But the argument dependency ' $H^{n+1}\left(F^{n+1}, F^{n}\right)$ ' is not type-theoretically acceptable. For the order of any defined relation must be higher than the orders of its arguments.

ANALYSIS 65.2, April 2005, pp. 000-000. (C) Jeffrey Ketland 
To make the definition type-theoretically acceptable, we might write instead,

(6) $\forall F^{n+1} \forall F^{n}\left(H^{n+2}\left(F^{n+1}, F^{n}\right) \leftrightarrow \sim F^{n+1}\left(F^{n}\right)\right)$.

This is a type-theoretically acceptable definition. But this does not lead to contradiction (at least, not obviously). For $\mathrm{H}^{n+2}$ is, by assumption, a type $n+2$ relation and thus lies outside the range of the initial quantifiers. Furthermore, $F^{n+1}$ is required to be a one-place relation, while $H^{n+2}$ is a two-place relation. It would be a category mistake to instantiate a variable for one-place relations with (a constant for) a two-place relation.

In short, what is wrong with Jacquette's argument is that (according to his own intentions) the defined heterologicality relation $H^{n+1}$ is a twoplace relation, with arguments $F^{n+1}$ and $F^{n}$. When this is clarified, the proposed definition (i.e. (5) above) violates type-restrictions.

The mistake here illustrates nicely a phenomenon in the theory of definitions, namely that the formulation of definitions must satisfy certain formal constraints. One of these constraints is that any free variable that appears in the definiens must also appear in the definiendum. ${ }^{1}$ Jacquette's 'definition' (1) violates this constraint.

Violating these constraints can lead to an amusing 'proof' that Peano arithmetic (indeed, any theory which proves that there are least two objects) is inconsistent. For example, assume the Peano axioms for arithmetic, and define a predicate ' $P$ ' as follows:

(7) $\forall x \forall y(P(x) \leftrightarrow x<y)$.

From (7), we can infer,

(8) $\forall y(P(1) \leftrightarrow 1<y)$.

Thus,

(9) $P(1) \leftrightarrow 1<2$.

And thus, since ' $1<2$ ' is provable,

(10) $P(1)$.

But also, from (7), we may infer,

(11) $P(1) \leftrightarrow 1<0$.

And thus, since ' $1<0$ ' is refutable,

(12) $\sim P(1)$.

1 See Haack 1978: 103-4 for a short discussion of these constraints in relation to Tarski's definition of truth. 
Does this remarkably simple argument show that arithmetic is inconsistent? No, it merely shows that 'definitions' like (7), and, similarly, Jacquette's (1), violate the aforementioned formal constraint.

University of Edinburgh Edinburgh EH8 9JX, UK jeffrey.ketland@ed.ac.uk

\section{References}

Haack, S. 1978. Philosophy of Logics. Cambridge: Cambridge University Press. Jacquette, D. 2004. Grelling's revenge. Analysis 64: 251-56. 